\title{
Hematopoietic stem cells can differentiate into restricted myeloid progenitors before cell division in mice
}

Tatyana Grinenko ${ }^{1}$, Anne Eugster ${ }^{2}$, Lars Thielecke ${ }^{3}$, Beáta Ramasz $^{1}$, Anja Krüger ${ }^{1}$, Sevina Dietz $^{2}$, Ingmar Glauche (10 3, Alexander Gerbaulet ${ }^{4}$, Malte von Bonin (10 5,6,7, Onur Basak ${ }^{8,9,10}$, Hans Clevers ${ }^{8,9,11}$, Triantafyllos Chavakis ${ }^{1,2}$ \& Ben Wielockx (D) 1,2

Hematopoietic stem cells (HSCs) continuously replenish all blood cell types through a series of differentiation steps and repeated cell divisions that involve the generation of lineage-committed progenitors. However, whether cell division in HSCs precedes differentiation is unclear. To this end, we used an HSC cell-tracing approach and Ki67 RFP knock-in mice, in a non-conditioned transplantation model, to assess divisional history, cell cycle progression, and differentiation of adult HSCs. Our results reveal that HSCs are able to differentiate into restricted progenitors, especially common myeloid, megakaryocyteerythroid and pre-megakaryocyte progenitors, without undergoing cell division and even before entering the $\mathrm{S}$ phase of the cell cycle. Additionally, the phenotype of the undivided but differentiated progenitors correlated with the expression of lineage-specific genes and loss of multipotency. Thus HSC fate decisions can be uncoupled from physical cell division. These results facilitate a better understanding of the mechanisms that control fate decisions in hematopoietic cells.

\footnotetext{
${ }^{1}$ Department of Clinical Pathobiochemistry, Institute for Clinical Chemistry and Laboratory Medicine, Technische Universität Dresden, Fetscherstraße 74, 01307 Dresden, Germany. ${ }^{2}$ DFG Research Centre and Cluster of Excellence for Regenerative Therapies Dresden, Technische Universität Dresden, Fetscherstraße 105, 01307 Dresden, Germany. ${ }^{3}$ Institute for Medical Informatics and Biometry (IMB), Technische Universität Dresden, Fetscherstraße 74, 01307 Dresden, Germany. ${ }^{4}$ Institute for Immunology, Technische Universität Dresden, Fetscherstraße 74, 01307 Dresden, Germany. ${ }^{5}$ Medical Clinic and Policlinic I, University Hospital Carl Gustav Carus, Technische Universität Dresden, Fetscherstraße 74, 01307 Dresden, Germany. ${ }^{6}$ German Cancer Consortium (DKTK), partner site Dresden, Fetscherstraße 74, 01307 Dresden, Germany. ${ }^{7}$ German Cancer Research Center (DKFZ), Im Neuenheimer Feld 280, 69120 Heidelberg, Germany. ${ }^{8}$ Hubrecht Institute, Royal Netherlands Academy of Arts and Sciences and University Medical Center Utrecht, Uppsalalaan 8, 3584 CT Utrecht, The Netherlands. ${ }^{9}$ Cancer Genomics Netherlands, UMC Utrecht, Heidelberglaan 100, 3584 CX Utrecht, The Netherlands. ${ }^{10}$ Department of Translational Neuroscience, Brain Center Rudolf Magnus, University Medical Center Utrecht and Utrecht University, 3584 CG Utrecht, The Netherlands. ${ }^{11}$ Princess Máxima Centre, Lundlaan 6, 3584 EA Utrecht, The Netherlands. Correspondence and requests for materials should be addressed to T.G. (email: tatyana.grinenko@uniklinikum-dresden.de) or to B.W. (email: ben.wielockx@tu-dresden.de)
} 
rare population of hematopoietic stem cells (HSCs) resides at the top of the hematopoietic hierarchy ${ }^{1}$. Although most adult HSCs normally exist in a quiescent or dormant state ${ }^{2}$, some of them divide and support the production of all mature blood cell types through multiple intermediate progenitor stages, during steady state, and in response to acute needs ${ }^{3-5}$. These include myeloid progenitors (MPs), encompassing restricted progenitors like common myeloid progenitors (CMPs), granulocyte-macrophage progenitors (GMPs), pre-megakaryocyte-erythroid progenitors (PreMEs), and pre-megakaryocyte progenitors (PreMegs). This classical point of view was questioned in recent studies from two groups showing that HSC populations contain stem-cell-like megakaryocyte progenitors, which under stress conditions such as transplantation into irradiated recipients ${ }^{6}$ or after acute inflammation ${ }^{7}$ activate a megakaryocyte differentiation program. The commitment process(es) that turns HSCs into mature cells are currently understood to be a sequence (or even a continuum) of decision steps in which the multilineage potential of the cells is sequentially $\operatorname{lost}^{8-10}$. Although many of these steps have been investigated in great detail, the entire picture is still repeatedly challenged $6,8,9,1$ P-13. HSC transition through the multipotent and restricted progenitor stages is also accompanied by intense cell proliferation ${ }^{3}$. However, it is unclear whether each fate decision step is associated with one or more division events or whether cell proliferation and differentiation are independent processes. Further, if differentiation of HSCs does require cell division, the phase of the cell cycle that is particularly important for this process is also currently unknown. The dependence of cell fate decisions on cell cycle progression was so far only shown in vitro for pluripotent embryonic stem cells ${ }^{14-17}$. However, a few reports point toward a functional connection between these two processes in adult stem cells, such as neuronal stem cells ${ }^{16,18}$. With regard to hematopoietic stem and progenitor cells, characterization of the cell cycle itself is currently ongoing ${ }^{19-22}$, and an understanding of how HSC fate decisions relate to cell division and cell cycle progression is lacking ${ }^{19}$.

Therefore, we used in vivo cell tracing to simultaneously follow the divisional history and the initial differentiation steps of HSCs. Our data reveal that HSCs are able to differentiate into restricted progenitors prior to cell division, most prominently PreMEs and PreMegs, and that this occurs before the cells enter the $S$ phase of the cell cycle. Moreover, our data also demonstrate that the G0/G1 phases are important for fate decision in HSCs to either differentiate or self-renew.

\section{Results}

HSCs differentiate into MPs without dividing. To study the initial steps of HSC differentiation in vivo, we sorted $\mathrm{Lin}^{-} \mathrm{Kit}^{+}$ $\mathrm{Sca}^{+} 1^{+}$(LSK) CD $48^{-}$CD $41^{-}$CD $150^{+}$stem cells (Fig. 1a) ${ }^{1}$. CD $41^{+}$ cells were excluded to reduce myeloid- ${ }^{23}$ and megakaryocyte-biased HSCs $^{24-26}$. We used the CellTrace Violet dye ${ }^{27,28}$ to uniformly label HSCs and track cell division history after transplantation (Fig. 1a). Recently, Shimoto et al. have shown that numerous empty HSC niches are available upon transplantation into nonconditioned recipients, which are located distant from filled niches and available for HSC engraftment and proliferation. Moreover, donor HSCs give rise to all blood cells without any bias $^{29}$. Labeled cells were transplanted into unconditioned recipients to prevent irradiation-induced stress $^{30-32}$ (Fig. 1a). Thirty-six hours after transplantation, $30 \%$ of the donor cells had downregulated Sca-1 expression (Fig. 1b), one of the principal surface marker for $\mathrm{HSCs}^{33}$, and changed their phenotype from HSCs to MPs. Importantly, the purification procedure alone did not lead to downregulation of Sca-1 (Supplementary Fig. 1a). A possible contamination of potential donor MPs was excluded, since transplantation of these progenitors alone did not result in any detectable donor MPs $36 \mathrm{~h}$ later (Supplementary Fig. 1b). To further classify these phenotypically restricted MPs in vivo, we used a gating strategy according to Pronk and colleagues ${ }^{34}$. Briefly, PreMEs (Lin ${ }^{-} \mathrm{Scal}^{-} \mathrm{Kit}^{+} \mathrm{CD}^{-} 1^{-} \mathrm{CD} 16 / 32^{-} \mathrm{CD} 105^{-}$ $\mathrm{CD}_{150}{ }^{+}$), Pre-CFU-E (Lin ${ }^{-} \mathrm{Scal}^{-} \mathrm{Kit}^{+} \mathrm{CD}^{-} 1^{-} \mathrm{CD} 16 / 32^{-}$ $\left.\mathrm{CD}_{105}{ }^{+} \mathrm{CD} 150^{+}\right)$, CFU-E (Lin ${ }^{-} \mathrm{Scal}^{-} \mathrm{Kit}^{+} \mathrm{CD} 41^{-} \mathrm{CD} 16 / 32^{-}$ $\left.\mathrm{CD}_{105^{+}} \mathrm{CD}_{150^{-}}\right)$, PreMegs $\left(\mathrm{Lin}^{-} \mathrm{Scal}^{-} \mathrm{Kit}^{+} \mathrm{CD} 41^{+}\right.$ $\mathrm{CD} 16 / 32^{-} \mathrm{CD}_{150}{ }^{+}$), and Pre-GM ( $\mathrm{Lin}^{-} \mathrm{Sca}^{-} \mathrm{Kit}^{+} \mathrm{CD} 41^{-}$ $\left.\mathrm{CD} 16 / 32^{-} \mathrm{CD} 105^{-} \mathrm{CD} 150^{-}\right)$staining was initially confirmed by transplantation of cells into lethally irradiated mice (Supplementary Fig. 2a, b). However, Pre-GMs gave rise to platelets and myeloid and erythroid cells after transplantation and were therefore classified as CMPs. Based on surface staining at 36 $\mathrm{h}$ posttransplantation, we subdivided donor MPs into the following restricted progenitors: CMPs, GMPs ( $\mathrm{Lin}^{-} \mathrm{Scal}^{-} \mathrm{Kit}^{+}$ CD41- ${ }^{-}$D $150^{-}$CD16/32 ${ }^{+}$), PreMEs, and PreMegs (Fig. 1b).

Next, we analyzed the proliferation history of transplanted cells based on dilution of CellTrace Violet dye, whereby intensity of the dye in $\mathrm{CD}_{4}^{+}{ }^{+} \mathrm{CD} 62 \mathrm{~L}^{+}$naive $\mathrm{T}$ cells was used as the reference for undivided cells (Supplementary Fig. 1c) ${ }^{35,36}$. This analysis reveals that, at $36 \mathrm{~h}$ after HSC transplantation, a majority of LSK cells with the long-term HSC phenotype (LSK CD $48^{-} \mathrm{CD} 150^{+}$), short-term HSCs (ST-HSCs) (LSK CD48- ${ }^{-} \mathrm{CD} 150^{-}$), multipotent progenitors (MPP2: LSK CD48 ${ }^{+} \mathrm{CD} 150^{+}$and MPP3/4 LSK $\left.\mathrm{CD} 48^{+} \mathrm{CD} 150^{-}\right)(\text {Supplementary Fig. } 3 \mathrm{a})^{1}$ and $50 \%$ of the MPs remained undivided (Fig. 1c). Additionally, based on CD41 and CD150 expression, these MPs were predominantly CMPs, PreMEs, and PreMegs (Fig. 1d, e). We also performed an even more stringent gating strategy to avoid overlay between nondivided and divided cells (Supplementary Fig. 1d) but found no difference in the frequency of restricted progenitors, as compared to the previous gating strategy (Supplementary Fig. 1e). To exclude the possibility that HSCs differentiated into MPs without division due to the limited niche space, we used the HSC-CreERT $+\mathrm{R} 26^{\mathrm{DTA} / \mathrm{DTA}}$ mouse line allowing for the inducible depletion of HSCs and transplanted CellTrace dye labeled wild-type HSCs into them ${ }^{37}$ (Supplementary Fig. 1f). However, we did not find any difference in the frequency of HSCs differentiated into myeloid-restricted progenitors $36 \mathrm{~h}$ after transplantation, compared to controls (Supplementary Fig. 1g, h). Surprisingly, compared to mice not preconditioned with tamoxifen (TAM), we found that donor HSCs in TAM-treated mice displayed enhanced differentiation into GMPs without cell division, suggesting potentially additional stress induced by TAM.

Interestingly, transplantation of MPP2 or MPP3/4 subsets revealed a similar phenomenon as most of the MPs did not divide. Further, while MPP2 cells mostly gave rise to PreMEs and PreMeg cells, MPP3/4 cells differentiated into CMPs and GMPs (Supplementary Fig. 3b-d). Taken together, these results strongly suggest that HSCs/MPPs can give rise to restricted progenitors including CMPs, PreMEs, and PreMegs based on the cell phenotype, without undergoing cell division.

Undivided differentiated progenitors express lineage genes. To investigate the molecular differences between undivided HSCs and undivided MPs, we designed a panel of primers to analyze single-cell expression levels of 70 genes including cell cycle genes and those specific for HSCs, myeloid, erythroid, megakaryocyteerythroid progenitors (MEP), and platelets (Supplementary Table 1$)^{8,911,12,33,38-41}$. Essentially, single-cell expression analysis of freshly sorted HSCs, CMPs, PreMEs, and PreMegs showed a clear separation of the cell types whether based on all analyzed 
a

Sort HSC (LSK CD41/48- CD150-)

+ CellTrace Violet labeling

$\longrightarrow$ FACS

Gating strategy
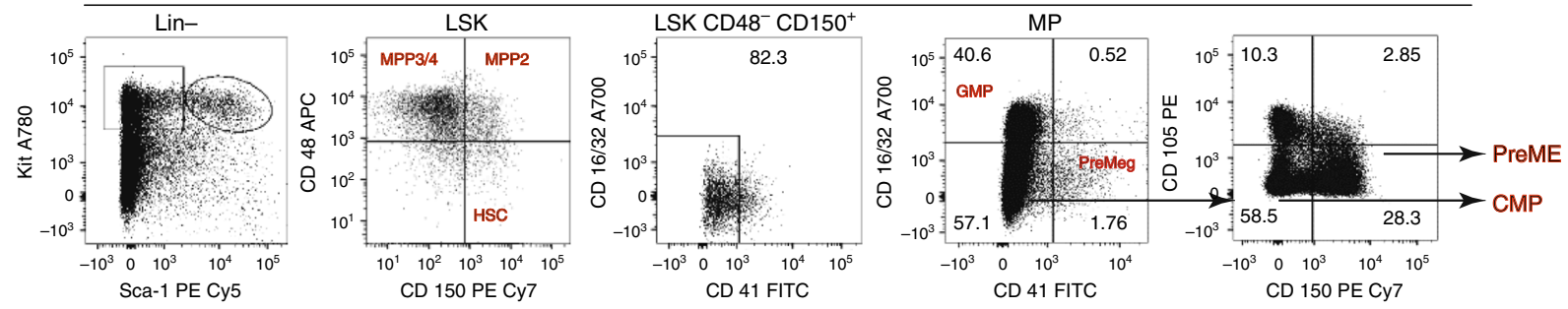

CD 41 FITC

CD 41 FITC

CD 150 PE Cy7

Transplanted cells

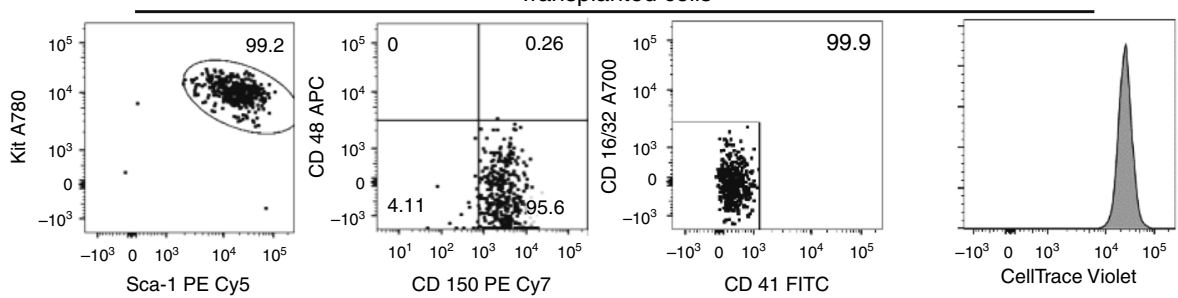

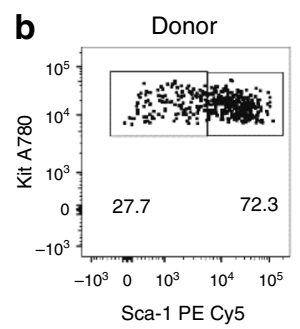

c

Donor LSK

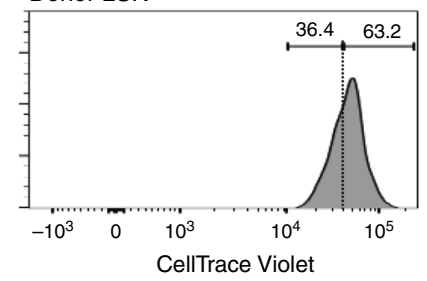

d
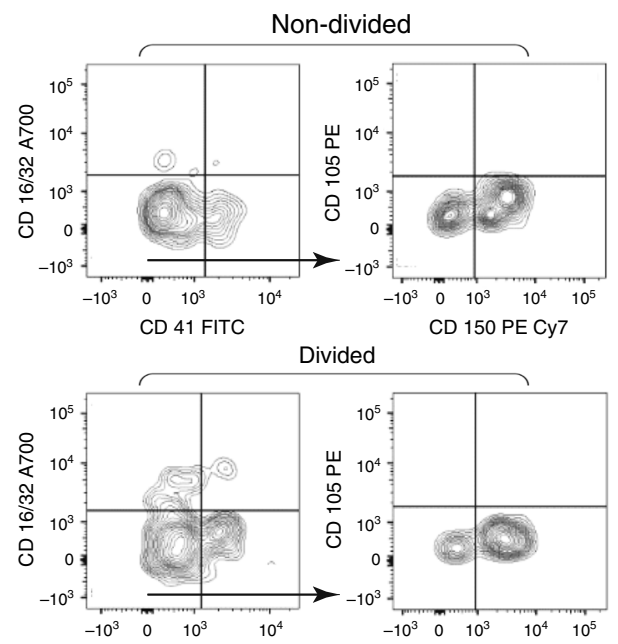

CD 41 FITC

Donor LSK

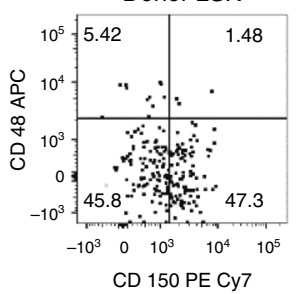

CD 150 PE Cy7

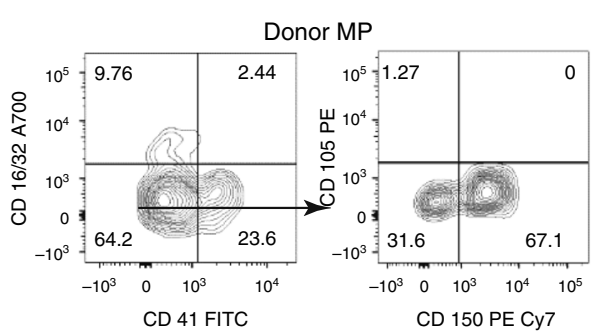

Donor MP

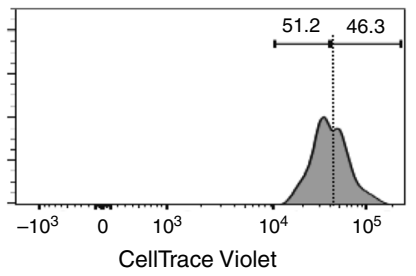

e

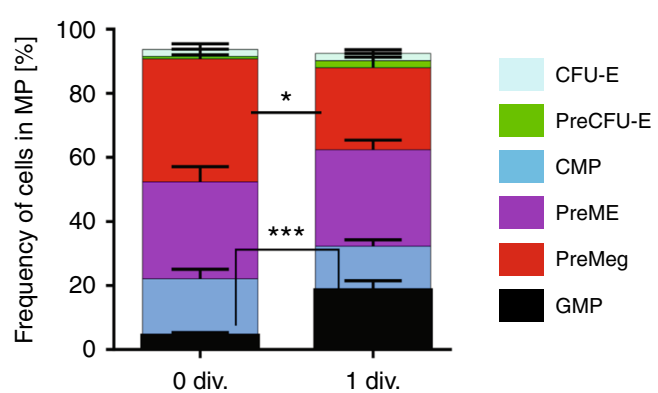

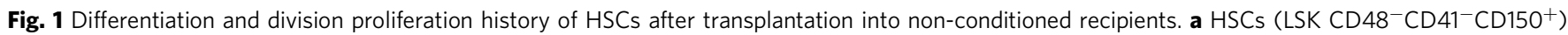
were labeled with CellTrace Violet dye and 3600 cells were transplanted into non-conditioned wild-type mice. Purity of transplanted cells was $>99 \%$ for each experiment. b Bone marrow was harvested at $36 \mathrm{~h}$ after transplantation and donor cells were analyzed using the indicated gates. c Dilution of CellTrace Violet in donor LSK and MPs, $36 \mathrm{~h}$ after transplantation. Labeled and transplanted naive CD62L ${ }^{+} \mathrm{CD} 4^{+} \mathrm{T}$ cells were used as reference for undivided cells. Five hundred donor cells were analyzed from 11 transplanted mice, representative data for 1 out of 13 experiments. d Phenotype of undivided and divided donor MPs $(n=11)$, representative example of 13 independent experiments. e Frequency of restricted progenitors in undivided ( 0 div.) and divided (1 div.) donor MPs, pooled data from 13 independent experiments. Unpaired Student's $t$-test, data are means $+/-$ s.d., ${ }^{\star \star \star} P=0.0002,{ }^{\star} P=0.02$ 
genes or only on selected $\mathrm{MEP} /$ platelet genes (Supplementary Fig. $4 \mathrm{a}-\mathrm{c})$.

We then isolated undivided donor cells at $36 \mathrm{~h}$ after transplantation of LSK CD $48^{-} \mathrm{CD}^{-} 1^{-} \mathrm{CD} 150^{+}$cells (Fig. 2a) and retrospectively categorized them on the basis of index sorting data as HSCs (LSK CD48 ${ }^{-} \mathrm{CD}^{-} 50^{+}$) or various MP populations (Supplementary Fig. 5a-d). Within these populations, we performed single-cell quantitative PCR (qPCR) on 42 HSCs, 7 CMPs, 15 PreMEs, and 20 PreMegs pooled and obtained from two independent experiments (Fig. 2b). Performing t-distributed

a

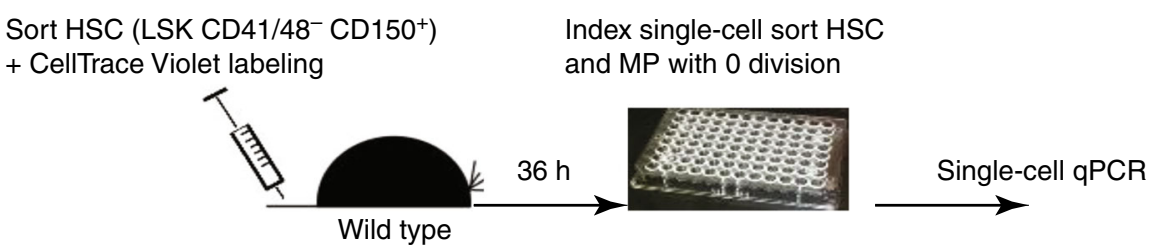

b

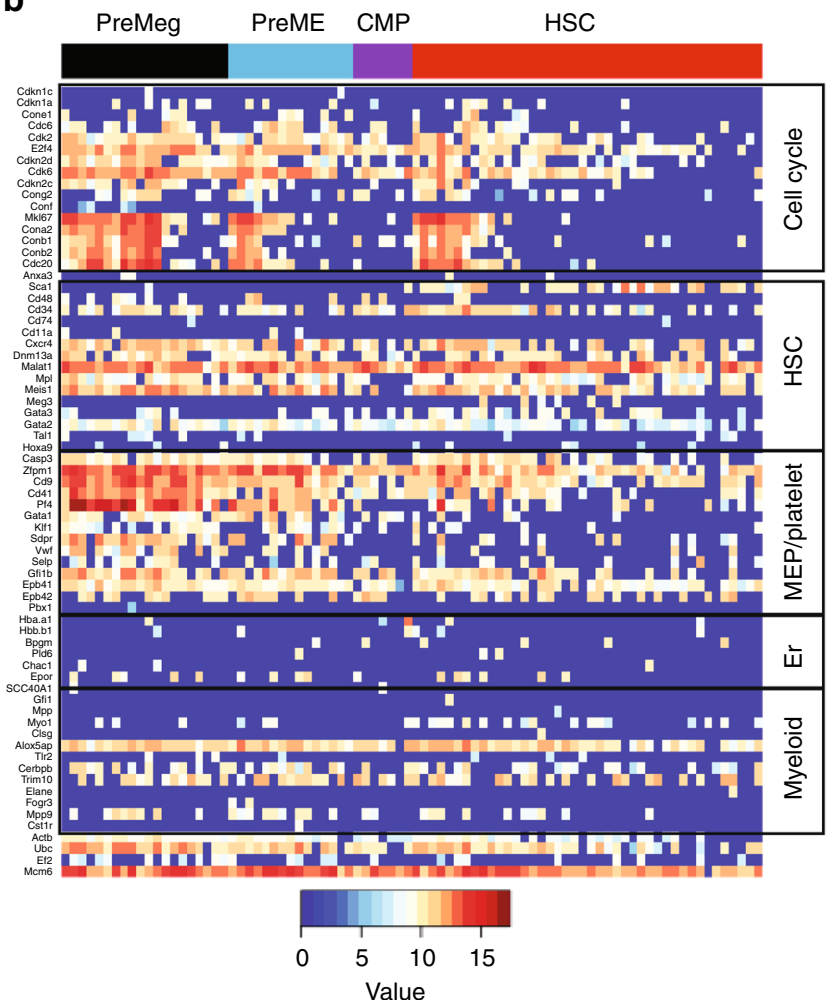

c

C All genes

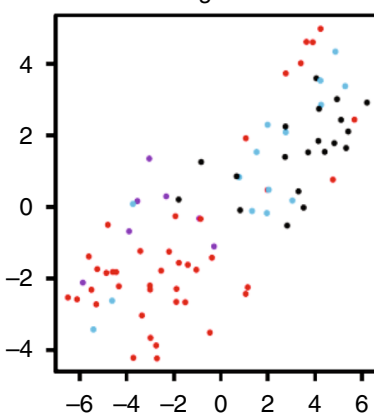

d

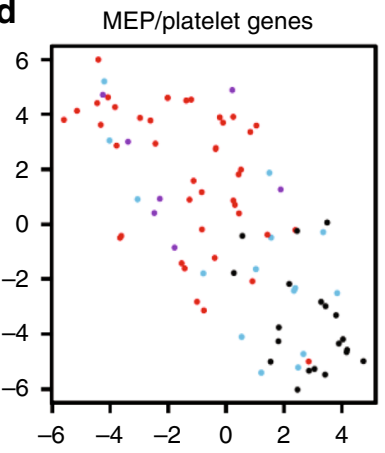

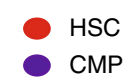

- PreME

PreMeg

e

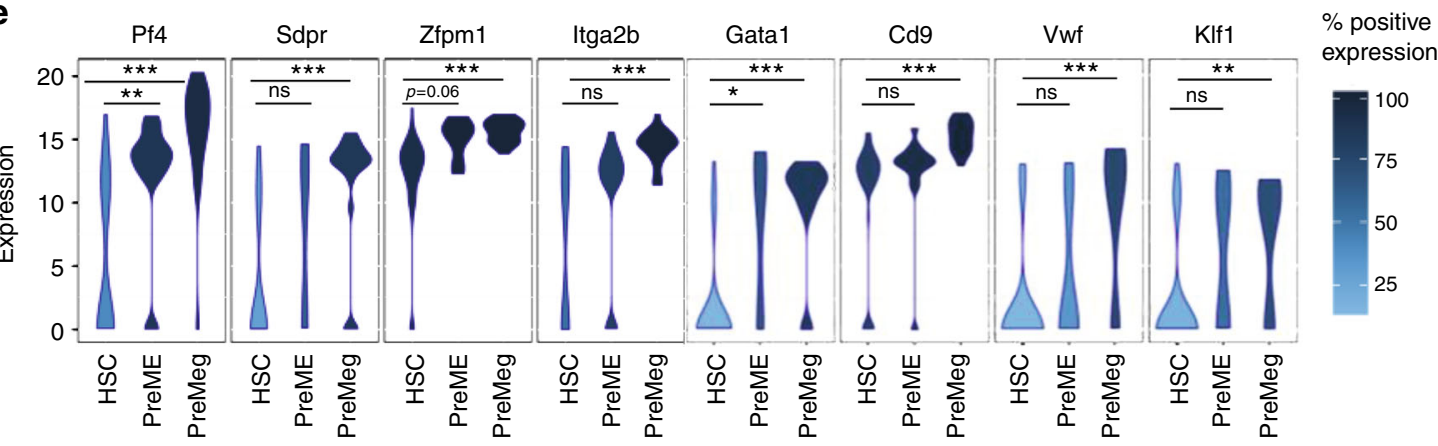

Fig. 2 Single-cell expression analysis in undivided donor HSCs and MPs. a Experimental design. LSK CD48- CD41- CD150 ${ }^{+}$cells were transplanted into non-irradiated recipients, and single, undivided donor $\mathrm{Lin}^{-} \mathrm{Kit}^{+}$cells were sorted using the index sort approach at $36 \mathrm{~h}$ after transplantation. Data from two independent experiments $\left(n=12\right.$ mice). Based on index sort data, HSCs were defined as LSK CD48- CD150 ${ }^{+}$; CMPs as $\mathrm{Lin}^{-} \mathrm{Kit}^{+} \mathrm{Sca}-1^{-} \mathrm{CD} 16 / 32^{-} \mathrm{CD}^{-} 1^{-}$

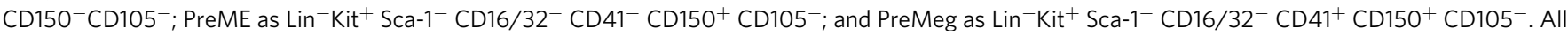
sorted 42 HSC, 7 CMP, 15 PreME, and 20 PreMeg cells were analyzed. $\mathbf{b}$ Heat map showing gene expression analysis. Each row corresponds to a specific gene, each column corresponds to a specific and individual donor cell, and colors represent the expression levels of individual genes (dCt). $\mathbf{c} t-S N E$ plot for all the analyzed genes and cells, axes display arbitrary units. d t-SNE plot for MEP/platelet genes for all cells, axes display arbitrary units. e Violin density plots for the most differently expressed MEP/platelet genes. y Axis represents gene expression. The horizontal width of the plot shows the density of the data along the $y$ axis. Statistical significance was determined using the Hurdle model. ${ }^{\star} P<0.05$; ${ }^{\star \star} P<0.01$; ${ }^{\star \star \star} P<0.0001$; ns, not significant. Data from two independent experiments, $n=12$. Exact $P$-value in supplemental Tables S2,3 
stochastic neighbor embedding (t-SNE) analysis of the qPCR data revealed separation of HSCs from PreMEs and PreMegs, based on all analyzed genes (Fig. 2c) or the MEP/platelet genes alone (Fig. 2d, Supplementary Table 1). This separation among phenotypically defined populations was also confirmed by a majority of the MEP/platelet-specific genes (Fig. 2e, Supplementary Tables $2,3,4,5$ ) and was similar to that observed before transplantation (Supplementary Fig. 4d). Thus undivided PreME/PreMeg cells obtained after transplantation express genes typically restricted to MEP.

For an in-depth comparative analysis of the transplanted undivided cells (Fig. 2) and non-transplanted cells (Supplementary Fig. 4), we performed $\mathrm{t}-\mathrm{SNE}^{42}$ and hierarchical cluster analysis on gene expression data (Fig. 3a, b, Supplementary Fig. 5e). We wondered whether HSCs and PreMegs truly form distinctive subgroups in terms of their gene expression profile. Therefore, we excluded the intermediate cell differentiation stages (colored in green) and provided the algorithm with a number of expected clusters $(k=2)$. Figure $3 \mathrm{~b}$ illustrates that not only the visual inspection of the $\mathrm{t}$-SNE visualization but also the $k$-means cluster algorithm is able to distinguish between those two cell types. As expected, while our results reveal a close association between the before- and after-transplantation HSC or PreMeg populations, HSCs and PreMegs themselves form distinct clusters. Therefore, changes in the HSC phenotype before cell division reflect gene expression changes associated with differentiation.

HSCs differentiate before the $S$ phase of the cell cycle. While the cell-tracing dye allowed us to follow cell division, it did not give information on cell cycle progression. Therefore, to determine in which phase of the cell cycle HSCs make fate decisions, we scored each cell for its likely cell cycle phase using signatures for G1 and $\mathrm{S} / \mathrm{G} 2 / \mathrm{M}$ phases ${ }^{39}$. We categorized individual cells in the G0/G1 or the S/G2/M phases (Fig. 4a) based on the average expression of phase-specific genes ${ }^{39,43}$. As expected, and later confirmed by expression of individual cell cycle genes (Fig. 4b), HSCs were more quiescent, with almost one third of the PreME/PreMeg cells still in the G0/G1 phases (Fig. 4a). We also confirmed cluster separation between cells in G0/G1 and S/G2/M phases by performing t-SNE analysis based on all 15 measured cell cycle genes but restricted to PreME/PreMeg populations (Fig. 4c). To determine whether the expression of $\mathrm{MEP} /$ platelet genes is dependent on progression through the S/G2/M phases, we again used t-SNE analysis to compare PreME/PreMeg cells in the G0/G1 and S/G2/ $\mathrm{M}$ phases. There was no separation of cells according to their cell cycle status (Fig. 4c), suggesting that PreME/PreMeg cells had previously upregulated differentiation genes in the G0/G1 phases of the cell cycle. That PreME and PreMeg cells increase the expression of lineage-specific genes independent of cell cycle phase was further supported by comparing the mean expression of MEP/platelet genes between cells in G0/G1 and S/G2/M phases (Fig. 4d). Indeed, PreME and PreMeg cells increase the expression of the lineage-specific genes independent of cell cycle phases. These data imply that transplanted HSCs are able to differentiate before entering the $\mathrm{S}$ phase of the cell cycle.

To corroborate these findings, we used $\mathrm{Ki} 67^{\mathrm{RFP}}$ knock-in mice ${ }^{44}$. KI67 is a nuclear protein that is absent in the G0 phase, starts to be synthesized at the beginning of the $S$ phase, increases until mitosis, and gradually decreases thereafter in the G1 phase of the daughter cells until re-entry into the $\mathrm{S}$ phase ${ }^{45}$. We first confirmed that none of the RFP ${ }^{-}$cells (LSK or MP) was in the $\mathrm{S} / \mathrm{G}_{2} / \mathrm{M}$ phase, (Supplementary Fig. 6a) and that only $\mathrm{RFP}^{+}$cells incorporated bromodeoxyuridine (BrdU; Supplementary Fig. 6b). Using an antibody against KI67, we found that $\mathrm{RFP}^{+}$expression truly reflects KI67 expression at the protein level (Supplementary Fig. 6c). Thus Ki67 ${ }^{\mathrm{RFP}}$ knock-in mice are an appropriate tool to trace cell cycle progression in hematopoietic cells.

To follow HSCs through cell cycle progression and differentiation, we sorted $\mathrm{RFP}^{-}$HSCs residing in the G0/G1 phases, labeled them with CellTrace Violet, and transplanted these cells into nonconditioned recipients. Our results reveal that the majority of donor undivided MPs did not upregulate RFP expression (Fig. 4e), thus remaining in the G0/G1 phases. When taken together with the above results, these findings demonstrate that phenotypic HSCs do not require S-phase entry to become phenotypic MPs.

Functional differences between undivided HSCs and progenitors. We used in vitro colony assays to verify functional differences between undivided phenotypic HSCs and MPs due to changes in gene expression profiles. Undivided donor HSCs (LSK $\mathrm{CD} 48^{-} \mathrm{CD} 150^{+}$) and PreMegs $\left(\mathrm{Lin}^{-} \mathrm{Sca}-1^{-} \mathrm{Kit}^{+} \mathrm{CD} 150^{+} \mathrm{CD} 41\right.$ ${ }^{+}$) were isolated at $36 \mathrm{~h}$ after transplantation and cultured as single cells in the presence of growth factors (stem cell factor (SCF), thrombopoietin, interleukin-3, and erythropoietin) ${ }^{46}$. Twelve days later, $89 \%$ of HSCs were multipotent and gave rise to all cell types (myeloid, erythroid, and megakaryocyte), whereas 92\% of the PreMegs differentiated into megakaryocytes alone, clearly suggesting that this population had lost their multipotency (Fig. 5a).

We further investigated the in vivo repopulating capacity of donor cells. For this, we sorted undivided donor $\mathrm{GFP}^{+} \mathrm{LSK}$ and MP cells obtained at $36 \mathrm{~h}$ after transplantation of LSK CD $48 / 41^{-}$ $\mathrm{CD} 150^{+}$cells, and re-transplanted the same amount of cells into
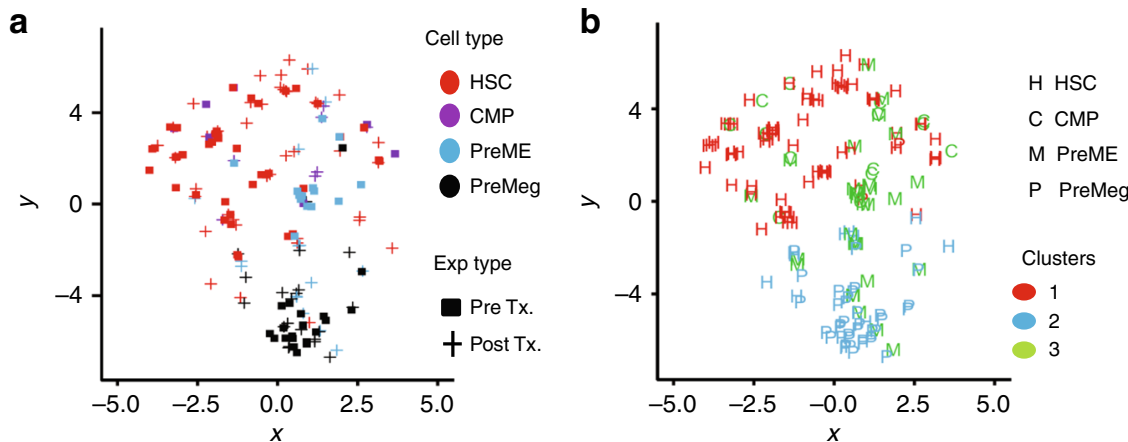

Fig. 3 Comparison of gene expression between cells before transplantation and undivided cells after transplantation. a t-SNE plot for MEP/platelet genes for all cells before transplantation and undivided donor cells at $36 \mathrm{~h}$ after transplantation. Axes display arbitrary units. $\mathbf{b}$ t-SNE visualization for all cells before transplantation and all undivided cells after transplantation $(36 \mathrm{~h})$. The color coding depicts the results of a reproducible $k$-means clustering $(k=2)$ on all cells before and after transplantation based on MEP/platelet genes 
a

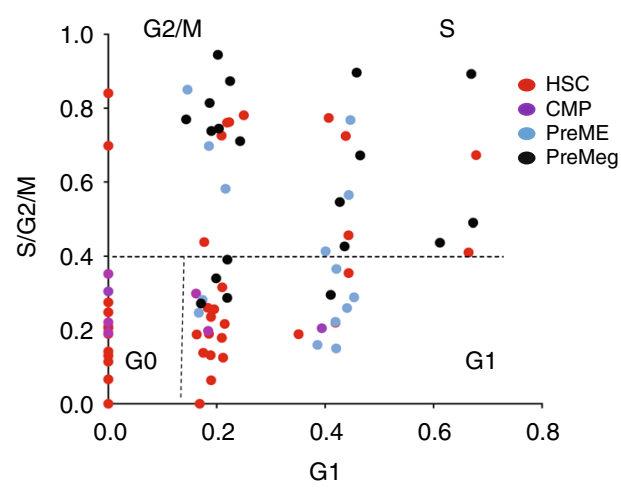

b

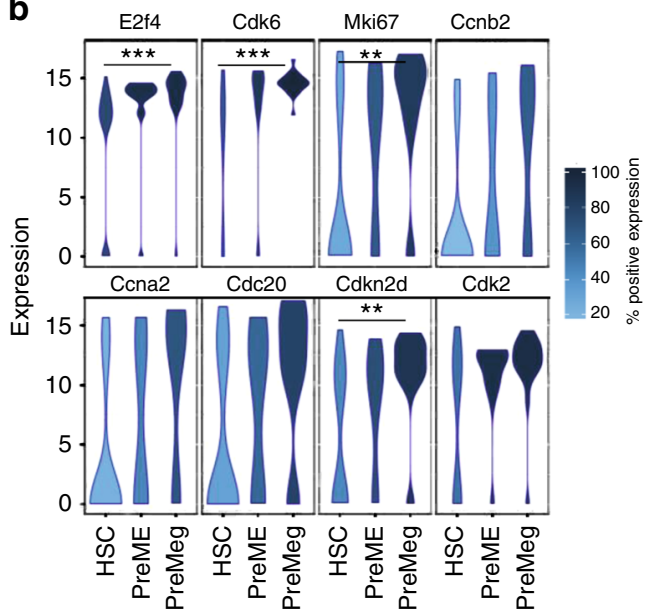

C
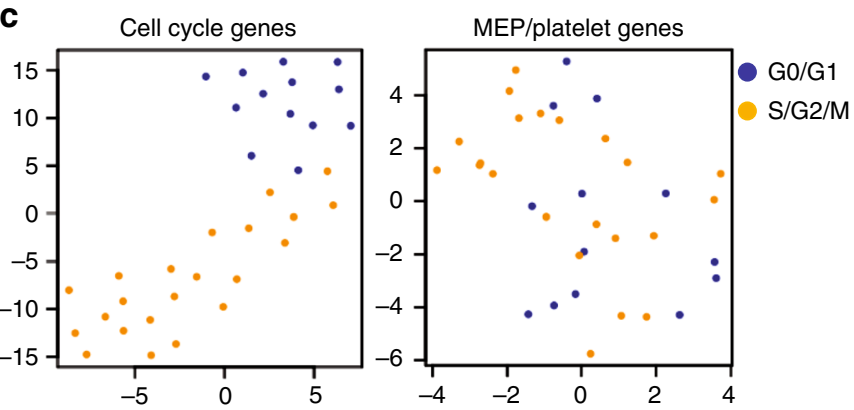

e

RFP $^{-}$HSCs from Ki67-RFP mouse (in G0/G1 phase) labeled with CellTrace Violet

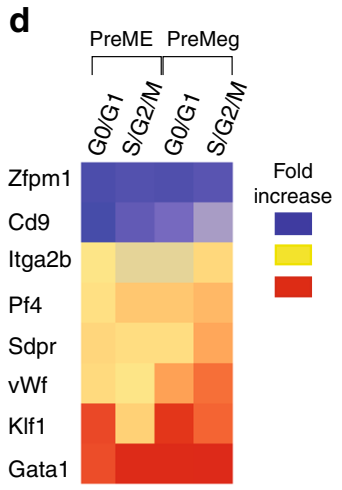

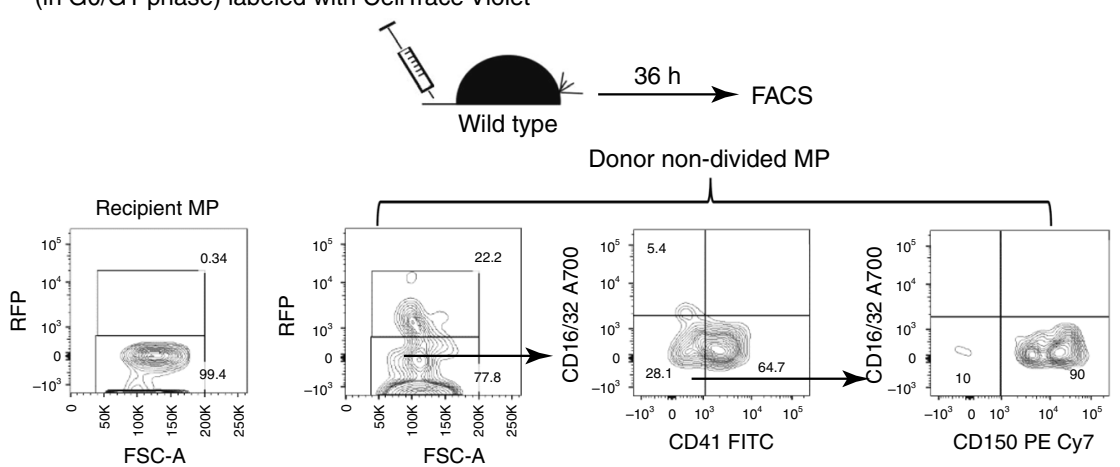

Fig. 4 Cell cycle distribution of undivided donor HSCs, CMPs, PreMEs, and PreMegs. a Prediction of cell cycle phases for all undivided donor cells $36 \mathrm{~h}$ after transplantation. Shown is the average expression of G1 genes ( $x$ axis) and S/G2/M genes ( $y$ axes). b Violin density plots for the most differently expressed cell cycle genes. y Axis represents gene expression. The horizontal width of the plot shows the density of the data along the $y$ axis. Statistical significance was determined using the Hurdle model. ${ }^{\star} P<0.05$, ${ }^{\star \star} P<0.01,{ }^{\star \star \star} P<0.0001$. Exact $P$-value in supplemental Tables $2-3$. c t-SNE plots for PreME/PreMeg cells based on cell cycle genes and MEP/platelet genes. d Mean expression of MEP/platelet genes was calculated for HSCs, PreMEs, and PreMegs in GO/ G1 and S/G2/M phases and is depicted as fold increase relative to mean expression in HSCs in the G0/G1 phases. e RFP expression in undivided donor MPs at $36 \mathrm{~h}$ after transplantation of RFP- $\mathrm{HSCs}$ from Ki67 RFP knock-in mice. Recipient MPs were used as negative controls for RFP expression. (representative example, $n=5$, from 2 independent experiments)

lethally irradiated wild-type mice (Fig. 5b, c). Although both populations gave rise to long-lived erythroid cells, only mice transplanted with LSKs displayed donor-derived $\mathrm{GFP}^{+}$ short-lived neutrophils and platelets at 3 weeks after transplantation (Fig. 5b-d); moreover, LSK but not MP showed donorderived neutrophils, platelets, erythrocytes, and lymphocytes 16 weeks after transplantation (Supplementary Fig. 6d). These observations imply that hematopoietic progenitor cells that downregulate Sca-1 without prior cell division, as expected, exhibit a dramatic reduction in their repopulation capacity.

\section{Discussion}

In this study, we demonstrated in vivo that HSCs can differentiate into ST-HSCs, MPPs, and even restricted MPs before undergoing cell division. Using a cell-tracing approach and Ki67 ${ }^{\mathrm{RFP}}$ knock-in mice, we followed HSC differentiation in vivo and analyzed the expression of several essential megakaryocyte-erythroid- and myeloid-specific genes, and cell cycle genes, at the single-cell level. Our findings using undivided PreMegs reveal that phenotypic and gene expression changes in undivided but differentiated progenitors are accompanied by loss of multipotency and repopulation capacity after transplantation. Based on restricted 
a HSCs labeled with
CellTrace Violet
$($ LSK CD48/41- CD150 $)$

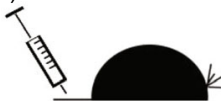

$36 \mathrm{~h}$ Single cell index sort non-divided HSC and PreMeg PreMeg
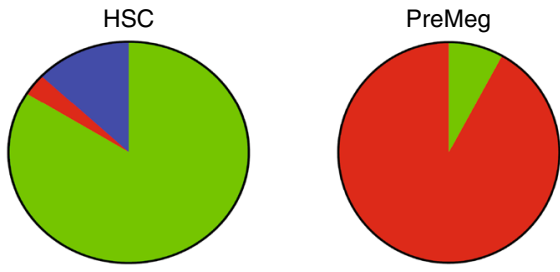

All cell types

Megakaryocytes

Myeloid cells

b

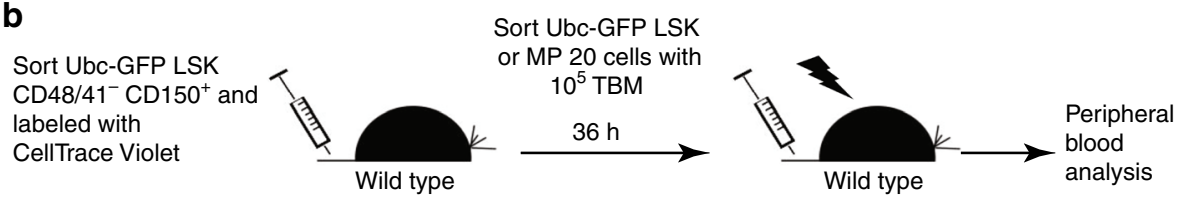

C
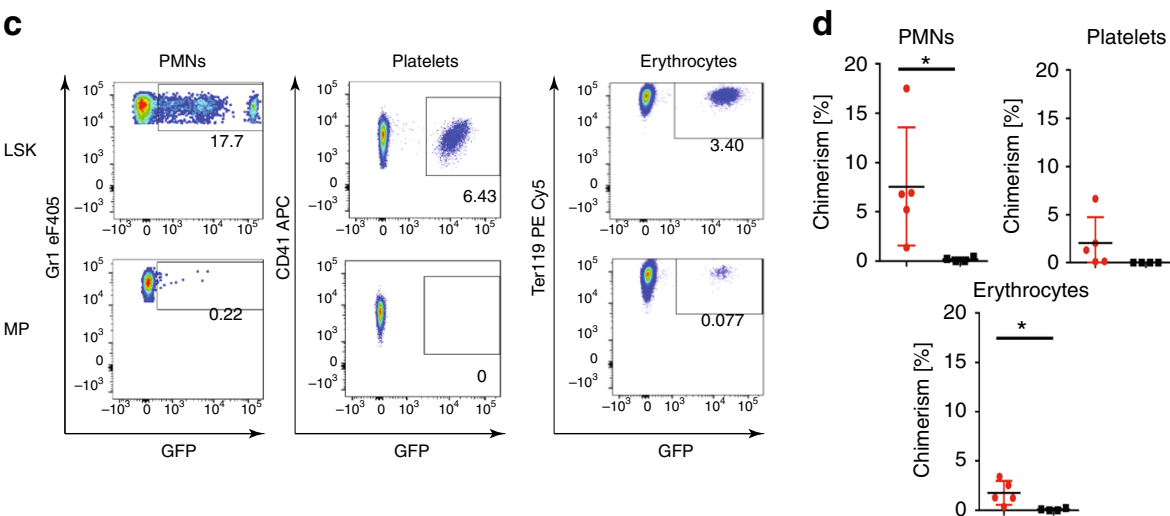

Fig. 5 Functional analysis of undivided donor HSCs and MPs. a Individual undivided donor HSCs (LSK CD48 ${ }^{-} \mathrm{CD}_{150^{+}}$) and PreMeg $\left(\mathrm{Lin}^{-} \mathrm{Sca}^{-} 1^{-} \mathrm{Kit}^{+}\right.$ $\mathrm{CD} 41^{+} \mathrm{CD}_{150^{+}} \mathrm{CD} 16 / 32^{-}$) cells were sorted $36 \mathrm{~h}$ after transplantation and cultivated in liquid culture media supplemented with mSCF, mTPO, mll3, and hEpo. Cell composition was analyzed after 12 days using May-Grunwald-Giemsa staining. Colonies $(n=31)$ for HSCs and $(n=25)$ PreMegs, 3 independent experiments, 15 mice. In all, 82\% HSCs generated colonies ( $>20$ cells) and 79\% PreMegs generated $>3$ megakaryocytes. b Reconstitution experiment using Ubc-GFP mice. c Peripheral blood analysis at 3 weeks after secondary transplantation into lethally irradiated recipients. Donor cell contribution to peripheral blood neutrophils (PMNs) $\mathrm{CD}_{11 \mathrm{~b}^{+}} \mathrm{Gr}^{+}$, platelets Ter119- $\mathrm{CD} 41^{+}$, and erythrocytes Ter119 ${ }^{+}$. Plots and pictures from 2 independent experiments $(n=5)$. LSK repopulation is shown in the upper panel and MP in the bottom panel. We checked the mice every 3-4 weeks for a period of 16 weeks after transplantation but did not find any repopulation from MPs. d Quantification of peripheral blood analysis 3 weeks after transplantation from 2 independent experiments, $n=5$. LSK is in red, MP is in black. Statistical significance was determined using unpaired Student's $t$-test $\left({ }^{\star} P<0.05\right)$. Data are means $+/-$ s.d.

PreME and PreMeg progenitors as an example of differentiated cells, we reveal that HSCs can initiate a specific differentiation program in the G0/G1 phases, which is before the actual physical division of the cell.

HSCs are rare cells that give rise to numerous blood cell types through a series of intermediate progenitors ${ }^{4}$. Multipotent and restricted progenitors intensively proliferate, making them the key amplifiers of cell numbers in the hematopoietic system ${ }^{3}$. The currently accepted model of hematopoiesis holds that HSCs have to divide in order to produce multipotent and lineage-restricted progenitor populations $3,47,48$. Thus, with respect to HSCs, proliferation and differentiation are currently characterized as simultaneous processes; however, to date, no direct in vivo proof of this concept has been provided. On the contrary, it is also conceivable that proliferation and differentiation exist as two independent processes. A few in vitro studies have supported this argument and have suggested that HSC division and differentiation are parallel processes. Indeed, while Mossadegh-Keller and colleagues ${ }^{49}$ have shown that the myeloid transcription factor PU.1 is induced during the first cell cycle after in vitro stimulation of HSCs with macrophage colony-stimulating factor, Yamamoto and colleagues ${ }^{6}$ reported that HSCs can divide asymmetrically and give rise to restricted long-term repopulating megakaryocyte progenitors even after the first division. Kent and colleagues ${ }^{50}$ have shown that HSCs downregulated a number of transcription factors responsible for self-renewal division and lost long-term repopulation capacity before first division in vitro. Using a singlecell sequencing approach, Yang and colleagues demonstrated that HSCs can express megakaryocyte and granulocyte-specific genes during the G1 phase of the cell cycle ${ }^{51}$. However, no in vivo studies on the possible uncoupling of HSC fate decision and cell cycle progression are currently available. 
Indeed, the idea that cells can make fate decisions in the G1 phase of the cell cycle is not new. Pluripotent stem cells (PSCs) initiate differentiation during progression through the G1 phase ${ }^{14}$ due to the presence of a 'window of opportunity', which is dependent on epigenetic changes that occur during that phase. On the other hand, PSCs maintain their pluripotent state during the $S$ and G2 phases of the cell cycle, which is regulated by the cell cycle machinery but is independent of the G1 phase ${ }^{17}$. G1-phasespecific cell cycle regulators such as cyclin D directly regulate the localization of differentiation transcriptional factors in $\mathrm{PSCs}^{52}$. Our results reveal a new avenue by which the HSC fate decision process is connected with cell cycle progression in vivo. Moreover, our data are also in line with another report, which demonstrated that division and differentiation of $\mathrm{B}$ cells into plasma cells were temporally separated with no significant influence on each other ${ }^{53}$.

In summary, we show that HSC division and their differentiation are probably independent processes and that HSCs make fate decisions before entering the $S$ phase of the cell cycle.

Additionally, these results open new directions in determining similar capacities in human HSCs, as well as identifying the factors that influence these fate decisions in connection with cell cycle progression, during normal hematopoiesis and even pathologies associated with abnormal differentiation.

\section{Methods}

Mice. C57BL/6 (B6), B6.SJL-PtprcaPep3b/BoyJ (B6.SJL), and Ubc:GFP mice were purchased from the Jackson Laboratory. Ki67 ${ }^{\mathrm{RFP}}$ knock-in mice have been recently described in detail ${ }^{44}$. Mice (male and female) were used at an age of 8-12 weeks. HSC-CreERT/R-DTA mice were generated by crossing R26 ${ }^{\text {DTA }}$ (Gt(ROSA)

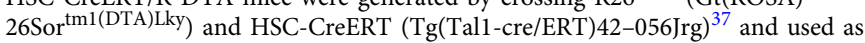
recipients (male and female) at the age of 14-16 weeks. All mice were bred and maintained under specific pathogen-free conditions in the animal facility at the Medical Theoretical Center of the University of Technology, Dresden. Experiments were performed in accordance with the German animal welfare legislation and were approved by the "Landesdirektion Sachsen - Referat 24.1".

Cre-activation. One week before the start of TAM administration, mice were kept on low phytoestrogen standard diet (LASvendi, Solingen, Germany). TAM tablets $30 \mathrm{mg}$ (Ratiopharm, Ulm, Germany) were dissolved overnight in lipid emulsion (SMOFlipid, Fresenius Kabi, Bad Homburg, Germany). TAM 20 mg/ml solution was applied two times ( $72 \mathrm{~h}$ apart) by oral gavage at a dose of $0.2 \mathrm{mg} / \mathrm{g}$ body weight to animals at the age of $8-14$ weeks.

Transplantation. Bone marrow (BM) was isolated from mouse tibia, femora, pelvis, and vertebrae; crushed; and filtered through a $70-\mu \mathrm{m}$ cell strainer. Cells were lysed in ACK Lysis Buffer (Life Technologies Cat. A10492-01) and lineage depleted using biotinylated antibodies (anti-mouse CD3 (2C11; 17A2) (1:1000/Cat. 130031-82), CD11b (M1/70) (1:500/Cat. 13-0112-81), CD19 (1D3) (1:500/Cat. 130193-81), CD45R (RA3-6B2) (1:400/Cat. 13-0452-82), Gr-1 (RB6-8C5) (1:800/Cat. 13-5931-82), Nk1.1 (PK136) (1:2000/Cat. 13-5941-81), Ter119 (1:200/Cat. MA517819 ), and anti-biotin micro-beads using magnetic cell separation (Miltenyi Biotec Germany Cat. 130-090-485). Cells were then stained with antibodies and CellTrace Violet dye (Molecular Probes Cat. C34557) according to the manufacturer's instructions. Cells were sorted on a fluorescence-activated cell sorter (FACS) Aria II or III (BD Bioscience). In all, $3600 \mathrm{HSCs}\left(\mathrm{Lin}^{-} \mathrm{Sca}-1^{+} \mathrm{Kit}^{+}\right.$(LSK) $\left.\mathrm{CD}^{-} 8^{-} \mathrm{CD} 41^{-} \mathrm{CD} 150^{+}\right), 5000 \mathrm{MPP} 2\left(\mathrm{LSK} \mathrm{CD} 48^{+} \mathrm{CD} 150^{+}\right.$), or $10,000 \mathrm{MPP} 3 / 4$ (LSK CD $48^{+} \mathrm{CD} 150^{-}$) cells were transplanted via intravenous injection into nonconditioned C57BL/6 mice. CD4 ${ }^{+} \mathrm{CD}_{2} \mathrm{~L}^{+}$naive $\mathrm{T}$ cells $\left(10^{6}\right)$, labeled with CellTrace Violet, were transplanted as controls for undivided cells. Lymph node donor cells were analyzed $36 \mathrm{~h}$ after transplantation along with LSK cells. For transplantation of cells from Ki67 ${ }^{\mathrm{RFP}}$ knock-in mice, $\mathrm{RFP}^{-}$cells were sorted and donor BM cells were analyzed $36 \mathrm{~h}$ after transplantation, based on CellTrace Violet staining. For competitive transplantation, $20 \mathrm{GFP}^{+}$LSK cells or MPs ( $\mathrm{Lin}^{-} \mathrm{Sca}-1$ $\mathrm{Kit}^{+}$) were sorted $36 \mathrm{~h}$ after a primary transplantation of 3600 HSCs from UbcGFP mice into unconditioned recipient C57BL/6 mice. LSKs and MPs were transplanted together with $10^{5}$ non-fractionated BM cells from B6.SJL mice into lethally irradiated ( $900 \mathrm{cGy}$ ) C57BL/6 wild-type recipients.

Flow cytometry. All analyses were done on FACS Aria II and Canto (BD Bioscience). The antibodies used for staining are mKi67 (1:100/Cat. 11-5698-82), CD117 (2B8) (1:600/Cat. 47-1171-80), Sca-1 (D7) (1:100/Cat. 15-5981-81), Ter119 (Ter119) (1:200/Cat. 15-5921-81), CD41 (MWReg30) (1:400 (fluorescein isothiocyanate (FITC))-1:800 (allophycocyanin)/Cat. 11-0411-82), CD105 (MJ7/ 18) (1:200/Cat. 12-1051-82), CD16/32 (93) (1:50/Cat. 56-0161), CD11b (M1/70) (1:1200/Cat. 12-0112-81), Gr-1 (RB6-8C5) (1:800/Cat. 48-5931), CD3e (1:200/Cat. 17-0031-82), and CD45R (1:400/Cat. 13-0452-82) all from eBioscience. CD48 (HM48-1) (1:300/Cat. 103411) and CD150 (TC15-12F1) (1:50/Cat. 115914) are from BioLegend.

Single-cell index sorting. Isolated cells were single-cell sorted into 8-well strips containing $5 \mu \mathrm{l}$ of phosphate-buffered saline. To record marker levels of each cell, the FACS Diva-7 "index sorting" function was activated during cell sorting. Using index sorting, single cells were sorted from the entire $\mathrm{Lin}^{-} \mathrm{Kit}^{+}$CellTrace Violet ${ }^{+}$ space, and the intensities of the CellTrace Violet, Kit, Sca-1, CD41, CD48, CD150, CD105, and CD16/32 FACS markers were recorded and linked to each cell's position.

Cytospins. Cells were spun onto object slides at $200 \times g$, dried, and stained with May-Grunwald and Giemsa solution (Sigma Aldrich).

In vitro culture. Single cells were sorted and cultured in 96-well plates in StemSpan SFEM medium (STEMCELL Technologies, Cat. 09600) supplemented with $20 \mathrm{ng} /$ $\mathrm{ml} \mathrm{rmSCF}$ (Peprotech, 250-03), $20 \mathrm{ng} / \mathrm{ml} \mathrm{rmTPO}$ (eBioscience, 34-8686-63), $20 \mathrm{ng} /$ $\mathrm{ml} \mathrm{rmIl3}$ (Peprotech, 213-13), and $5 \mathrm{U} / \mathrm{ml} \mathrm{rhEpo} \mathrm{(Roche)} \mathrm{and} \mathrm{cultivated} \mathrm{for}$ 12 days at $37^{\circ} \mathrm{C}$ with $5 \% \mathrm{CO}_{2}$.

Cell cycle analyses. For intracellular staining, cells were fixed and permeabilized using fixation and permeabilization buffers from eBioscience. To distinguish between the G0 and G1 phase, cells were stained with intracellular Ki67 FITC (eBioscience, clone SolA15). DAPI (4, 6 diamidino-2-phenylindole; Molecular Probes) was used to measure DNA content and separate the cells in S/G2/M phases from those in the G0 and G1 phase. For the BrdU incorporation assay, $10 \mu \mathrm{M}$ BrdU (Sigma-Aldrich) was added to the culture for $3.5 \mathrm{~h}$ and BrdU incorporation analyses were performed using anti-BrdU-FITC ab (eBioscience, clone BU20a, Cat. \# $11-5071-42)^{46}$

Clustering-based analysis of cell cycle state. Cell cycle genes were classified based on single-cell deep sequencing data ${ }^{39}$ or defined previously in synchronized HeLa cells ${ }^{43}$ (G1 phase genes: Ccne1, Cdk2, Cdkn1a, Cdkn1c; S/G2/M phase genes: Cdkn2d, E2f4, Cdk6, Cdkn2c, Ccng2, Ccnf, Mki67, Ccna2, Ccnb1, Ccnb2, Cdc20). First, expression of each gene for each cell was normalized to the maximum expression of the gene; second, cell cycle signature for each cell was defined as the average expression of phase-specific subsets of cell cycle genes. Discrimination between $\mathrm{G} 1$ and S/G2/M was done based on the distribution of control HSCs (before transplantation) and data that around 90\% of HSCs (mouse strain C57Bl6) are in G0/G1 phase of the cell cycle.

Single-cell qPCR. Gene expression profiles of single cells were obtained using a modified protocol ${ }^{54,55}$. Briefly, cDNA was synthesized directly on the cells using the Quanta $\mathrm{qS}$ Cript ${ }^{\mathrm{TM}} \mathrm{cDNA}$ Supermix. Total cDNA was pre-amplified for 20 cycles $\left(1 \times 95^{\circ} \mathrm{C} 5^{\prime}, 95^{\circ} \mathrm{C} 45^{\prime \prime}, 60^{\circ} \mathrm{C}, 1^{\prime}, 72^{\circ} \mathrm{C} 1.5^{\prime}\right)$ and once at $68^{\circ} \mathrm{C}$ for $10^{\prime}$ using the Multiplex PCR Kit (Qiagen, Hilden, Germany) in a final volume of $35 \mu$ in the presence of primer pairs ( $25 \mathrm{nM}$ for each primer) for all genes (listed in Table S1). Pre-amplified cDNA $(10 \mu \mathrm{l})$ was then treated with $1.2 \mathrm{U}$ Exonuclease I, and gene expression was quantified by real-time PCR on the BioMark ${ }^{\mathrm{mm}}$ HD System (C) Fluidigm Corporation, CA, USA) using the 96.96 Dynamic Array IFC, the GE $96 \times$ 96 Fast PCR + Melt protocol, the SsoFast EvaGreen Supermix with Low ROX (BIO $\mathrm{RAD}, \mathrm{CA}, \mathrm{USA}$ ), and $5 \mu \mathrm{M}$ primers, for each assay. Raw data were analyzed using the Fluidigm Real-Time PCR analysis software.

Bioinformatics analysis. Pre-processing and data analysis of single-cell expression profiles were conducted using the KNIME 2.11.2, R Version 3.3.2, and RStudio Version 0.99.486 and version 1.0.136 (Boston, MA, USA) software. Where further required, pre-processing via a linear model to correct for confounding sampling effects was conducted ${ }^{54}$. t-SNE plots were created using the R package "Rtsne". To model the bi-modal gene expression of single cells, the Hurdle model, a semi-continuous modeling framework, was applied to pre-processed data ${ }^{56}$. This allowed us to assess differential expression profiles as a function of frequency of expression and mean positive expression using a likelihood ratio test. $k$-means clustering for $k=2$ was performed on the normalized data and using the R package "stats".

Statistical analysis. Data were expressed as mean $+/-$ standard deviation (s.d.) Statistical analyses based on unpaired Student's $t$-test were performed using the Prism 5.0 software (GraphPad). $P$-value $<0.05$ were considered as statistically significant. 
Data availability. All data generated or analyzed during this study are included in this published article and its Supplementary Information files or are available from the corresponding authors upon reasonable request.

Received: 2 June 2017 Accepted: 10 April 2018

Published online: 15 May 2018

\section{References}

1. Oguro, H., Ding, L. \& Morrison, S. J. SLAM family markers resolve functionally distinct subpopulations of hematopoietic stem cells and multipotent progenitors. Cell Stem Cell 13, 102-116 (2013).

2. Wilson, A. et al. Hematopoietic stem cells reversibly switch from dormancy to self-renewal during homeostasis and repair. Cell 135, 1118-1129 (2008).

3. Busch, K. et al. Fundamental properties of unperturbed haematopoiesis from stem cells in vivo. Nature 518, 542-546 (2015).

4. Sawai, C. M. et al. Hematopoietic stem cells are the major source of multilineage hematopoiesis in adult animals. Immunity 45, 597-609 (2016).

5. Nestorowa, S. et al. A single-cell resolution map of mouse hematopoietic stem and progenitor cell differentiation. Blood 128, e20-e31 (2016).

6. Yamamoto, R. et al. Clonal analysis unveils self-renewing lineage-restricted progenitors generated directly from hematopoietic stem cells. Cell $\mathbf{1 5 4}$ 1112-1126 (2013).

7. Haas, S. et al. Inflammation-induced emergency megakaryopoiesis driven by hematopoietic stem cell-like megakaryocyte progenitors. Cell Stem Cell 17, 422-434 (2015).

8. Hoppe, P. S. et al. Early myeloid lineage choice is not initiated by random PU.1 to GATA1 protein ratios. Nature 535, 299-302 (2016).

9. Paul, F. et al. Transcriptional heterogeneity and lineage commitment in myeloid progenitors. Cell 163, 1663-1677 (2015).

10. Velten, L. et al. Human haematopoietic stem cell lineage commitment is a continuous process. Nat. Cell Biol. 19, 271-281 (2017).

11. Cabezas-Wallscheid, N. et al. Identification of regulatory networks in HSCs and their immediate progeny via integrated proteome, transcriptome, and DNA methylome analysis. Cell Stem Cell 15, 507-522 (2014).

12. Guo, G. et al. Mapping cellular hierarchy by single-cell analysis of the cell surface repertoire. Cell Stem Cell 13, 492-505 (2013).

13. Haghverdi, L., Buettner, F. \& Theis, F. J. Diffusion maps for high-dimensional single-cell analysis of differentiation data. Bioinformatics 31, 2989-2998 (2015).

14. Pauklin, S. \& Vallier, L. The cell-cycle state of stem cells determines cell fate propensity. Cell 155, 135-147 (2013).

15. Singh, A. M. et al. Cell-cycle control of bivalent epigenetic domains regulates the exit from pluripotency. Stem Cell Rep. 5, 323-336 (2015).

16. Dalton, S. Linking the cell cycle to cell fate decisions. Trends Cell Biol. 25, 592-600 (2015).

17. Gonzales, K. A. et al. Deterministic restriction on pluripotent state dissolution by cell-cycle pathways. Cell 162, 564-579 (2015).

18. Lange, C., Huttner, W. B. \& Calegari, F. Cdk4/cyclinD1 overexpression in neural stem cells shortens G1, delays neurogenesis, and promotes the generation and expansion of basal progenitors. Cell Stem Cell 5, 320-331 (2009).

19. Laurenti, E. et al. CDK6 levels regulate quiescence exit in human hematopoietic stem cells. Cell Stem Cell 16, 302-313 (2015).

20. Mende, N. et al. CCND1-CDK4-mediated cell cycle progression provides a competitive advantage for human hematopoietic stem cells in vivo. J. Exp. Med. 212, 1171-1183 (2015).

21. Matsumoto, A. et al. p57 is required for quiescence and maintenance of adult hematopoietic stem cells. Cell Stem Cell 9, 262-271 (2011).

22. Zou, P. et al. p57(Kip2) and p27(Kip1) cooperate to maintain hematopoietic stem cell quiescence through interactions with Hsc70. Cell Stem Cell 9, 247-p261 (2011)

23. Gekas, C. \& Graf, T. CD41 expression marks myeloid-biased adult hematopoietic stem cells and increases with age. Blood 121, 4463-4472 (2013).

24. Miyawaki, K. et al. CD41 marks the initial myelo-erythroid lineage specification in adult mouse hematopoiesis: redefinition of murine common myeloid progenitor. Stem Cells 33, 976-987 (2015).

25. Roch, A., Trachsel, V. \& Lutolf, M. P. Brief report: single-cell analysis reveals cell division-independent emergence of megakaryocytes from phenotypic hematopoietic stem cells. Stem Cells 33, 3152-3157 (2015).

26. Bernitz, J. M., Kim, H. S., MacArthur, B., Sieburg, H. \& Moore, K. Hematopoietic stem cells count and remember self-renewal divisions. Cell 167, 1296-1309 (2016). e1210.
27. Pina, C. et al. Inferring rules of lineage commitment in haematopoiesis. Nat. Cell Biol. 14, 287-294 (2012).

28. Quah, B. J. C. \& Parish, C. R. New and improved methods for measuring lymphocyte proliferation in vitro and in vivo using CFSE-like fluorescent dyes. J. Immunol. Methods 379, 1-14 (2012).

29. Shimoto, M., Sugiyama, T. \& Nagasawa, T. Numerous niches for hematopoietic stem cells remain empty during homeostasis. Blood 129, 2124-2131 (2017)

30. Cao, X. et al. Irradiation induces bone injury by damaging bone marrow microenvironment for stem cells. Proc. Natl. Acad. Sci. USA 108, 1609-1614 (2011).

31. Abbuehl, J. P., Tatarova, Z., Held, W. \& Huelsken, J. Long-term engraftment of primary bone marrow stromal cells repairs niche damage and improves hematopoietic stem cell transplantation. Cell Stem Cell 21, 241-255 (2017). e246.

32. Schaue, D., Kachikwu, E. L. \& McBride, W. H. Cytokines in radiobiological responses: a review. Radiat. Res. 178, 505-523 (2012).

33. Wilson, N. K. et al. Combined single-cell functional and gene expression analysis resolves heterogeneity within stem cell populations. Cell Stem Cell 16, 712-724 (2015).

34. Pronk, C. J. et al. Elucidation of the phenotypic, functional, and molecular topography of a myeloerythroid progenitor cell hierarchy. Cell Stem Cell 1, 428-442 (2007).

35. Takizawa, H., Regoes, R. R., Boddupalli, C. S., Bonhoeffer, S. \& Manz, M. G. Dynamic variation in cycling of hematopoietic stem cells in steady state and inflammation. J. Exp. Med. 208, 273-284 (2010).

36. Pietras, E. M. et al. Chronic interleukin-1 exposure drives haematopoietic stem cells towards precocious myeloid differentiation at the expense of self-renewal. Nat. Cell Biol. 18, 607-618 (2016).

37. Schoedel, K. B. et al. The bulk of the hematopoietic stem cell population is dispensable for murine steady-state and stress hematopoiesis. Blood 128, 2285-2296 (2016).

38. Gottgens, B. Regulatory network control of blood stem cells. Blood 125, 2614-2620 (2015)

39. Kowalczyk, M. S. et al. Single-cell RNA-seq reveals changes in cell cycle and differentiation programs upon aging of hematopoietic stem cells. Genome Res. 25, 1860-1872 (2015).

40. Klimmeck, D. et al. Transcriptome-wide profiling and posttranscriptional analysis of hematopoietic stem/progenitor cell differentiation toward myeloid commitment. Stem Cell Rep. 3, 858-875 (2014).

41. Moignard, V. et al. Characterization of transcriptional networks in blood stem and progenitor cells using high-throughput single-cell gene expression analysis. Nat. Cell Biol. 15, 363-372 (2013).

42. van der Maaten, L. \& Hinton, G. Visualizing data using t-SNE. J. Mach. Learn. Res. 9, 2579-2605 (2008)

43. Whitfield, M. L. et al. Identification of genes periodically expressed in the human cell cycle and their expression in tumors. Mol. Biol. Cell 13, 1977-2000 (2002).

44. Basak, O. et al. Mapping early fate determination in Lgr5+crypt stem cells using a novel Ki67-RFP allele. EMBO J. 33, 2057-2068 (2014).

45. Scholzen, T. \& Gerdes, J. The Ki-67 protein: from the known and the unknown. J. Cell. Physiol. 182, 311-322 (2000).

46. Grinenko, T. et al. Clonal expansion capacity defines two consecutive developmental stages of long-term hematopoietic stem cells. J. Exp. Med. 211, 209-215 (2014)

47. Eaves, C. J. Hematopoietic stem cells: concepts, definitions, and the new reality. Blood 125, 2605-2613 (2015).

48. Sun, J. et al. Clonal dynamics of native haematopoiesis. Nature 514, 322-327 (2014).

49. Mossadegh-Keller, N. et al. M-CSF instructs myeloid lineage fate in single haematopoietic stem cells. Nature 497, 239-243 (2013).

50. Kent, D. G., Dykstra, B. J., Cheyne, J., Ma, E. \& Eaves, C. J. Steel factor coordinately regulates the molecular signature and biologic function of hematopoietic stem cells. Blood 112, 560-567 (2008).

51. Yang, J. et al. Single cell transcriptomics reveals unanticipated features of early hematopoietic precursors. Nucleic Acids Res. 45, 1281-1296 (2017).

52. Singh, A. M. et al. Signaling network crosstalk in human pluripotent cells: a Smad2/3-regulated switch that controls the balance between self-renewal and differentiation. Cell Stem Cell 10, 312-326 (2012).

53. Duffy, K. R. et al. Activation-induced B cell fates are selected by intracellular stochastic competition. Science 335, 338-341 (2012).

54. Bonifacio, E. et al. Effects of high-dose oral insulin on immune responses in children at high risk for type 1 diabetes: the Pre-POINT randomized clinical trial. JAMA 313, 1541-1549 (2015).

55. Fuchs, Y. F. et al. CD8+T cells specific for the islet autoantigen IGRP are restricted in their T cell receptor chain usage. Sci. Rep. 7, 44661 (2017). 
56. McDavid, A. et al. Modeling bi-modality improves characterization of cell cycle on gene expression in single cells. PLoS Comput. Biol. 10, e1003696 (2014).

\section{Acknowledgements}

T.G. received support from the Fritz Thyssen foundation (10.14.2.153). B.W. was supported by the Heisenberg program (Deutsche Forschungsgemeinschaft - DFG, Germany; WI3291/ 5-1). This work was supported by grants from the DFG (SFB655 "Cells into Tissues" to T.C. and GR4857/1-1 to T.G.), a CRTD seed grant to T.G. and B.W., and a European Research Council grant (683145) to T.C. The work of L.T. and I.G. was supported by the German Federal Ministry of Research and Education, Grant number 031A315 "MessAge". We would like to thank Dr. Vasuprada Iyengar for critically reading the manuscript.

\section{Author Contributions}

Conceptualization: T.G. and B.W.; investigation: A.K., S.D., and T.G.; methodology: T.G., A.E., L.T., B.R., M.v.B., A.G., and I.G.; resources: T.G., O.B., H.C., and B.W.; writing original draft and creating figures: T.G. and B.W.; writing, review, and editing: T.G., A.E, I.G., L.T., O.B., T.C., and B.W.; funding acquisition: T.G., L.T., I.G., T.C., and B.W.; supervision: T.G., T.C., and B.W.

\section{Additional information}

Supplementary Information accompanies this paper at https://doi.org/10.1038/s41467018-04188-7.
Competing interests: The authors declare no competing interests.

Reprints and permission information is available online at http://npg.nature.com/ reprintsandpermissions/

Publisher's note: Springer Nature remains neutral with regard to jurisdictional claims in published maps and institutional affiliations.

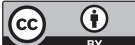

Open Access This article is licensed under a Creative Commons Attribution 4.0 International License, which permits use, sharing, adaptation, distribution and reproduction in any medium or format, as long as you give appropriate credit to the original author(s) and the source, provide a link to the Creative Commons license, and indicate if changes were made. The images or other third party material in this article are included in the article's Creative Commons license, unless indicated otherwise in a credit line to the material. If material is not included in the article's Creative Commons license and your intended use is not permitted by statutory regulation or exceeds the permitted use, you will need to obtain permission directly from the copyright holder. To view a copy of this license, visit http://creativecommons.org/ licenses/by/4.0/.

(C) The Author(s) 2018 